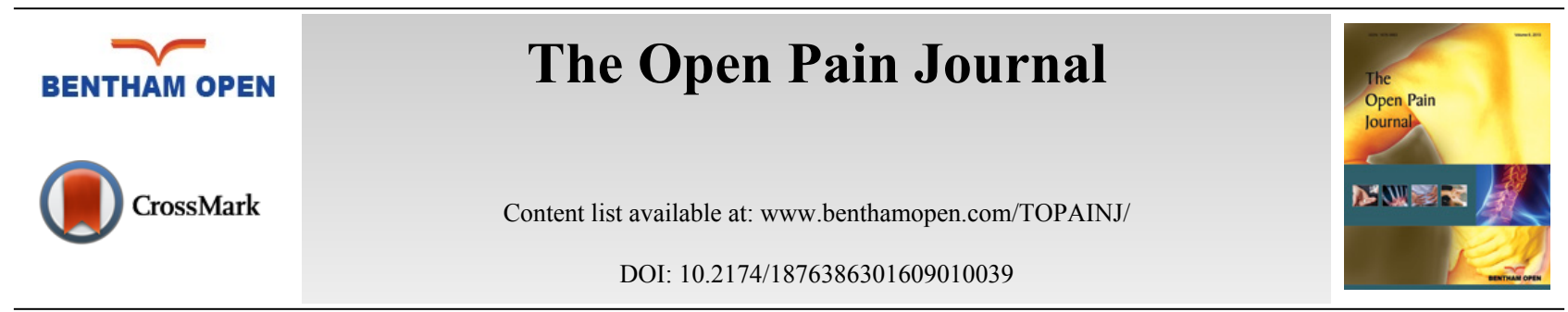

BOOK REVIEW

\title{
Surgical Pain Management
}

\author{
Serge Marchand ${ }^{*}$ \\ Department of Surgery, Department of Anaesthesia, Faculty of Medicine, Universite de Sherbrooke, Clinical Pain \\ Research Laboratories of the Research Centre of the Sherbrooke University Hospital, Sherbrooke, Quebec, Canada
}

Surgical approaches for pain were based on the specificity theory, suggesting specific pathways for nociceptive and non-nociceptive afferences. Surgical lesions of nociceptive pathways at different levels from the periphery to the central nervous system were used to interrupt the signal. In theory, selective lesions of nociceptive pathways should have completely blocked the nociceptive signal and the related pain perception. Unfortunately, we know that a lesion of the somatosensory pathways might not reduce the nociceptive signal and could even increase it and produce complex problems such as anesthesia dolorosa, pain in a deafferented territory. With the evolution of our understanding of the neurophysiology of pain, implantable neurostimulators or drug delivery systems are now the most popular surgical approaches.

With the collaboration of experts in the field of surgical approaches to pain management, Dr Narang, Dr Weisheipl and Dr Ross cover the most important surgical pain management approaches in a comprehensive and practical manner. They cover the important points to understand functional surgical approaches such as peripheral nerve stimulation, dorsal column stimulation, implantable drug delivery systems and deep brain stimulation.

In the first part of their book the authors emphasize the importance of patient selection and education to get the best results with surgical procedures that are generally end-of-line interventions for patients who failed to respond to conventional treatments.

Every section of the book is concise and practical. It is easy to find specific information on surgical procedures, anesthetic approaches for patients undergoing these surgeries and the importance of communication with the patient.

With the evolution of our understanding of the factors implicated in pain, surgical pain management is becoming more precise and more complex. More than ever, it requires a close collaboration between all health professionals implicated in pain management to really work together as a multidisciplinary team.

Any physician or health professional working in the field of chronic pain should have this book in their collection to better understand how implantable devices for the management of pain work and what the clinician and patient should know about them.

\footnotetext{
* Address correspondence to this author at the Department of Surgery, Department of Anaesthesia, Faculty of Medicine, Université de Sherbrooke Clinical Pain Research Laboratories of the Research Centre of the Sherbrooke University Hospital, Sherbrooke, Quebec, Canada; Tel: 1819 574-1229; E-mail: Serge.Marchand@USherbrooke.ca

(C) Narang et al.; Licensee Bentham Open

This is an open access article licensed under the terms of the Creative Commons Attribution-Non-Commercial 4.0 International Public License (CC BY-NC 4.0) (https://creativecommons.org/licenses/by-nc/4.0/legalcode), which permits unrestricted, non-commercial use, distribution and reproduction in any medium, provided the work is properly cited.
} 